\title{
Systematic review and meta-analysis: influence of iron deficiency anemia on blood glycosylated hemoglobin in diabetic patients
}

\author{
Ling Kuang ${ }^{1,2 \#}$, Wenxian $\mathrm{Li}^{3 \#}$, Guochao $\mathrm{Xu}^{4}$, Min You ${ }^{3}$, Wenqiao $\mathrm{Wu}^{5}$, Chenglong $\mathrm{Li}^{2,6}$ \\ ${ }^{1}$ Operation Department, Sichuan Provincial People's Hospital, University of Electronic Science and Technology of China, Chengdu, China; ${ }^{2}$ Chinese \\ Academy of Sciences Sichuan Translational Medicine Research Hospital, Chengdu, China; ${ }^{3}$ Stem Cell and Regenerative Medicine Research Center, \\ Sichuan Neo-Life Stem Cell Biotech Inc., Chengdu, China; ${ }^{4}$ Sichuan Umbilical Blood Bank, Sichuan Neo-Life Stem Cell Biotech Inc., Chengdu, \\ China; ${ }^{5}$ Flow Cytometry Room, Chengdu Neo-Life Hope Medical Laboratory Inc., Chengdu, China; ${ }^{6}$ Department of Hematology, Sichuan \\ Provincial People's Hospital, University of Electronic Science and Technology of China, Chengdu, China \\ Contributions: (I) Conception and design: L Kuang, W Li, C Li; (II) Administrative support: C Li; (III) Provision of study materials or patients: W Li, \\ G Xu, M You, W Wu; (IV) Collection and assembly of data: All authors; (V) Data analysis and interpretation: All authors; (VI) Manuscript writing: \\ All authors; (VII) Final approval of manuscript: All authors. \\ \#These authors contributed equally to this work. \\ Correspondence to: Chenglong Li. Department of Hematology, Sichuan Provincial People's Hospital, University of Electronic Science and Technology \\ of China, 32 West Section 2, First Ring Road, Qingyang District, Chengdu 610072, China. Email: lichenglong84@163.com.
}

Background: Diabetes is a common metabolic disease with an increasing incidence in middle-aged and
elderly people in recent years. Chronic hyperglycemia is the basic feature of diabetes, which can cause long-
term damage to eyes, kidneys, nerves, heart, and blood vessels, resulting in functional decline or even failure.
Glycosylated hemoglobin (HbAlc) can be used as an indicator of an individual's blood sugar status over the
past 3 months; however, it is slightly affected by ischemic anemia.

Methods: The data retrieval was performed in the databases of PubMed, Embase, and Ovid-Medline from their inception to April 2021, including keywords such as iron deficiency anemia (IDA), diabetes, HbA1c, immunoassay, and ion-exchange chromatography. After passing of sensitivity and heterogeneity analysis, Review Manager 5.3 was employed for meta-analysis.

Results: A total of 6 studies were included in this paper. The analysis results showed that IDA could be considered to have an impact on HbA1c outcomes in non-diabetic populations. In people with diabetes, IDA is not thought to have an impact on HbA1c outcomes.

Discussion: A total of 6 articles were included to discuss the effects of IDA on blood HbA1c in diabetic patients. The study found that when patients with diabetes were tested for blood sugar, the HbA1c did not accurately reflect their blood sugar control over the past 3 months.

Keywords: Iron deficiency anemia (IDA); glycosylated hemoglobin (HbA1c); immune method; iron exchange separation; meta-analysis

Submitted Sep 09, 2021. Accepted for publication Nov 09, 2021.

doi: 10.21037/apm-21-2944

View this article at: https://dx.doi.org/10.21037/apm-21-2944

\section{Introduction}

Diabetes mellitus (DM) is a syndrome of metabolic disorder caused by various influencing pathogenic factors, resulting in islet dysfunction, insulin resistance (IR), and so on, resulting in sugar, protein, fat, water, and electrolyte imbalance (1). The etiology may be related to genetic factors, immune dysfunction, microbial infection and its toxins, among others $(2,3)$. Chronic hyperglycemia is a basic feature of diabetes which can cause long-term damage to the eyes, kidneys, nerves, heart, and blood vessels, resulting in their compromised function and even failure 
$(4,5)$. According to the World Health Organization (WHO), the number of people with diabetes has nearly quadrupled in the last 40 years (6). Complications of diabetes can lead to heart attack, stroke, blindness, kidney failure, and amputation (7). Diabetes directly caused 1.5 million deaths in 2012. Therefore, accurate detection and strict control of blood sugar have become a very important and urgent matter (8).

Glycosylated hemoglobin (HbAlc) is a hemoglobin ( $\mathrm{Hb})$ composed of glycosylation of valine residues at the $\mathrm{NH}_{2}$ end of globin $\beta$ chain, which can be used as an indicator of blood glucose status in patients over the past 3 months $(9,10)$. Currently, HbA1c is widely accepted as a mean glycemic index, a measure of the risk of diabetes complications, and a measure of the quality of diabetes care (11). The measurement of HbAlc is attractive for diagnostic use because the single number provides a comprehensive assessment of glycemic control levels; however, it has some inherent limitations. Several factors unrelated to blood sugar can falsely decrease or increase HbAlc test results $(12,13)$. In addition, any factor that reduces the mean life expectancy of red blood cells, regardless of the assay used, mistakenly reduces the test results for HbAlc (14).

Iron deficiency anemia (IDA) is caused by the insufficient intake or excessive loss of iron in the body. Iron storage in the body is reduced when $\mathrm{Hb}$ appears, which can be of great harm to health (15). Abnormal iron metabolism is related to organ diseases such as liver and heart (16). The latest research suggests that abnormal iron metabolism plays an important role in the pathogenesis of IR and diabetes (17). An anemic state in which serum iron and blood cell indicators are normal is called a potential iron deficiency state $(18,19)$. Excessive iron load can damage pancreatic $\beta$ cells, trigger peripheral IR, and promote the occurrence of diabetes and its complications (20). The phlebotomy therapy to reduce iron load and the use of iron chelating agents can reduce IR, reduce glycated $\mathrm{Hb}$, improve abnormal blood lipid metabolism, and delay the occurrence and development of diabetes complications (21). At present, there is no uniform conclusion on the changes of serum HbA1c levels in IDA patients. Studies have shown that serum HbA1c levels in patients with diabetes and IDA are remarkably lower than healthy people (22). In nondiabetic people, IDA can increase serum HbA1c levels $(23,24)$. Of course, studies have confirmed that IDA has no considerable correlation with serum HbA1c levels (25).

In order to explore the correlation between IDA and HbA1c, provide more powerful information for defining the relationship between the two. By strictly evaluating and analyzing the existing case-control studies on the effect of IDA on HbA1c, this paper aimed to evaluate whether IDA can elevate HbA1c, so as to provide a basis for reasonable clinical evaluation of HbA1c in IDA patients.

We present the following article in accordance with the Preferred Reporting Items for Systematic Reviews and Meta-Analyses (PRISMA) reporting checklist (available at https://dx.doi.org/10.21037/apm-21-2944).

\section{Methods}

\section{Literature search strategy}

A comprehensive and systematic literature search was conducted based on the Cochrane Handbook of Systematic Reviews of Interventions (https://training.cochrane. org/handbook), with reporting meta-analyses following PRISMA. We searched the database of PubMed, PubMed, Embase, and Ovid-Medline, scientific conferences, and established articles. All the studies included patients with diabetes. Keywords and medical headings used in specific searches included the following: "iron deficiency anemia (IDA)", "iron deficiency", "iron therapy", "iron supplementation", or "HbA1c". The articles included were related to IDA and diabetes.

\section{Inclusion and exclusion criteria for articles}

Articles were included in the meta-analysis if they met the following inclusion criteria: (I) the study population was non-pregnant adults over 18 years of age; (II) the case group consisted of patients with a definite diagnosis of IDA who had not received iron therapy; (III) controls were healthy individuals without IDA or patients with other diseases that did not affect the study; (IV) study type: case-control study or cohort study; (V) the literature was complete or the data required for analysis could be extrapolated from the data given; (VI) English language, regardless of location.

Articles were excluded from the meta-analysis if they met any of the following exclusion criteria: (I) the study population included children under 18 years of age and pregnant women; (II) studies without control groups or cross-sectional studies; (III) secondary studies such as reviews; (IV) studies that were repeatedly published, of poor quality, inconsistent in study type, with too little information, and for which there was a lack of available data; (V) data prior to 1990 (data prior to 1990 were not 
included due to considerable differences in the methods used to measure HbA1c before and after 1990).

\section{Literature screening}

The titles and abstracts were firstly screen by two researchers independently according to the inclusion criteria, followed by data extraction and quality evaluation. If there was inconsistency of evaluation between the researchers, they consulted with other researchers to further resolve the discrepancy according to the literature and original data. When the title and abstract met the literature requirements, the full text was retrieved for data extraction. Note express 2.0 was used for literature management and deletion of duplicate literature. The inclusion of literature was conducted according to the inclusion and exclusion criteria mentioned above and relevant literature was searched.

\section{Data extraction}

The two researchers independently extracted relevant information from all eligible studies using a predefined data extraction table: author, year of publication, sample size, age, country, gender, degree of disease, and course of disease. In the case of missing data, the researchers attempted to contact the original authors of the literature via email. If the data were not available, the Cochrane evaluation manual was used for relevant interpretation, such as the calculation of standard deviation of continuous data.

\section{Quality assessment}

In order to improve the quality of the reviewed literature, the quality was assessed in accordance with the "risk of bias assessment" recommended in version 5.3 of the Cochrane Systematic Review Handbook. The evaluation includes the following seven items: (I) which random method was used; (II) whether allocation concealment was used; (III) implementation of the blind method between participants and researchers; (IV) evaluation of the effect of the blind method; (V) risk of bias assessment of the randomized controlled trial (RCT), "meet" indicates that bias was small "not satisfied" referred to high risk of bias, the study did not include a fully detailed report, if there was no mention of random sequence generation, allocation concealment, blinding, the risk was classified as unknown. A score of 1-3 in the 4 dimensions of tracking/exit was considered low quality, and a score of 4-7 was considered high quality.

\section{Data analysis}

A forest plot was drawn to present each individual study's results, combining those articles with corresponding confidence intervals (CIs). After no overlap was suggested among CIs of the included articles, there was certain statistical inhomogeneity among these articles. In further subgroup analysis, it was necessary that the stochastic and fixed models were combined with acceptable inhomogeneity. Subgroups were assigned regarding different designs, then the impact size of which was able to be overlooked. When the inhomogeneity among studies could not be ignored when different properties were investigated, some different properties were excluded from analysis in order to address the inhomogeneity.

Sensitivity analysis was conducted, which aimed to address if individual studies affected the overall results of the portfolio. Each study was removed one at a time. The results of each study were compared with the individual results to confirm whether the results were the same based on the results of the remaining studies. Generally speaking, it was found that an individual study impacted the comprehensive study in the following two circumstances. The presumption of the size of the combined effect was $95 \%$ of the size of the combined effect if a study was deleted. The results yielded remarkably different results after the deletion of one study. The sensitivity of the combined results was not stable if one study affected the overall results with only limited difference. In the opposite circumstances, the sensitivity was stable and the conclusion was correct.

\section{Statistical analysis}

Review Manager 5.3 (provided by the Cochrane Collaboration) was used for data processing in this systematic review, and the test level was 0.05 . When heterogeneity $\left(\mathrm{I}^{2}\right)<50 \%$ and $\mathrm{P}>0.05$, no statistical heterogeneity was determined among trials, and the fixed effects model was selected for meta-analysis. When $\mathrm{I}^{2} \geq 50 \%$ and $\mathrm{P} \leq 0.05$, statistical heterogeneity was considered among trials, and the random effects model was selected for meta-analysis. The combined effect size of the two groups of evaluation index data was odds ratio (OR) value and its $95 \% \mathrm{CI}$, and the forest map was drawn according to the integrated system evaluation results to display the research conclusions. The results with high heterogeneity were analyzed by eliminating articles one by one to explore the possible sources of heterogeneity, and 


\section{Identification of studies via databases and registers}

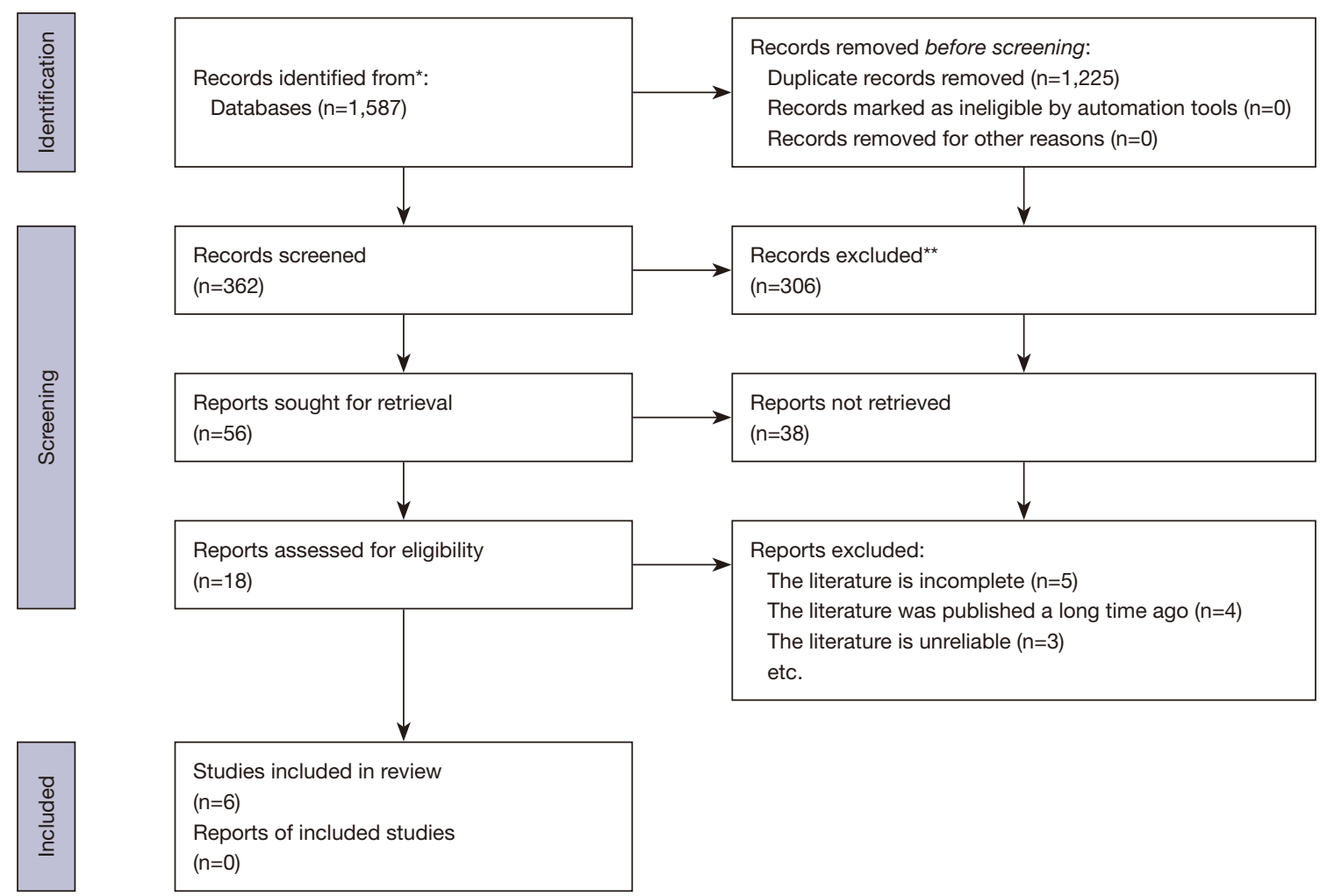

Figure 1 Literature retrieval process. *, consider, if feasible to do so, reporting the number of records identified from each database or register searched (rather than the total number across all databases/registers); **, if automation tools were used, indicate how many records were excluded by a human and how many were excluded by automation tools.

the sensitivity analysis was performed on the results.

\section{Results}

\section{Literature search results}

A total of 1,587 relevant articles were retrieved in this study, of which 984 were retrieved from PubMed, 545 from Embase, and 303 from Ovid-Medline database. After reading of titles and abstracts, 306 articles that clearly did not meet the inclusion criteria were excluded. After reading of full texts, 50 articles were excluded, and 6 articles (26-31) that met the inclusion criteria were finally included (Figure 1, Table 1).

\section{Bias-risk assessment of included articles}

The Cochrane Handbook (version 5.0.2) of the systematic review writing manual was used to evaluate the risk of bias in the 8 articles included in this study. Review Manager 5.3 was employed to output the risk of bias chart (Figures 2,3).

The Newcastle-Ottawa scale (NOS) was used to evaluate the quality of each included article, and the results are shown in Table 2. It can be seen that the 8 articles included in the study all had a low risk of bias, which met the requirements of subsequent analysis.

\section{Meta-analysis of the influence of IDA on $\mathrm{Hb}$ in diabetic patients}

A total of 4 articles met the requirements. For the index of serum $\mathrm{Hb}\left(\mathrm{P}<0.00001 ; \mathrm{I}^{2}=93 \%\right)$ in diabetic patients with IDA, data were combined between studies using the random effects model. The results showed that the experimental group was comparable to the control group. The difference in $\mathrm{Hb}$ content [mean difference $(\mathrm{MD})=-2.00 ; 95 \% \mathrm{CI}$ : 
Table 1 Basic characteristics of the included literature

\begin{tabular}{|c|c|c|c|c|c|c|c|}
\hline First author & $\begin{array}{c}\text { Published } \\
\text { year }\end{array}$ & $\begin{array}{c}\text { Experimental } \\
\text { group }\end{array}$ & $\begin{array}{l}\text { Control } \\
\text { group }\end{array}$ & $\begin{array}{c}\text { Study } \\
\text { population }\end{array}$ & Age (years) & $\mathrm{Hb}(\mathrm{g} / \mathrm{dL})$ & $\begin{array}{c}\text { Detection } \\
\text { method }\end{array}$ \\
\hline Coban E (26) & 2004 & 50 & 50 & Non-DM & $35.7 \pm 11.9$ & $10.8 \pm 1.2$ & IT \\
\hline Silva JF (28) & 2016 & 61 & 61 & Non-DM & $48 \pm 14 / 49 \pm 14$ & $13.2 \pm 1.1 / 9.4 \pm 1.9$ & HPLC, IT \\
\hline Christy AL (30) & 2014 & 70 & 50 & DM & $55.46 \pm 11.66$ & $12.54 \pm 1.4$ & HPLC \\
\hline Urrechaga E (31) & 2018 & 410 & 357 & DM & $>18$ & Unable to extract & HPLC \\
\hline
\end{tabular}

$\mathrm{Hb}$, hemoglobin; DM, diabetes mellitus; T1DM, type I diabetes mellitus; IT, immunization; HPLC, high performance liquid chromatography.

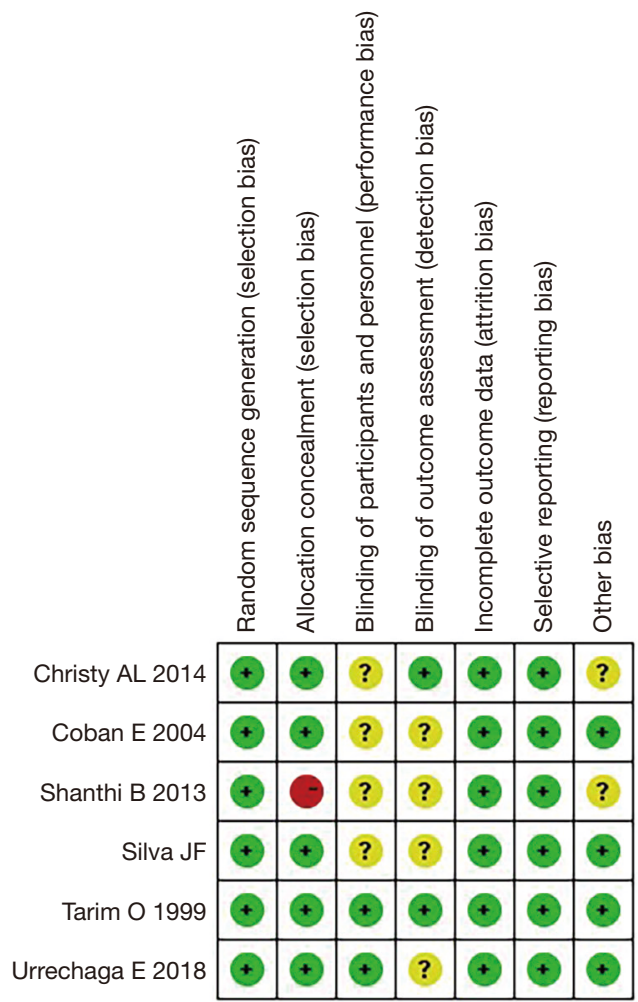

Figure 2 The bias-risk assessment diagram of the included articles.

-2.86 to $-1.14 ; \mathrm{P}<0.00001]$ between the two groups was statistically substantial, as shown in Figure 4.

Using the serum $\mathrm{Hb}$ of diabetic patients with IDA as the index, the results of the inverted funnel plot showed that the scattered points of the participants were roughly funneled downward. On the horizontal axis, they were arranged roughly symmetrically. Thus, it was suggested that publication bias was not obvious, as shown in Figure 5.

\section{Meta-analysis of the influence of IDA on $\mathrm{HbA1c}$ in diabetic patients}

A total of 6 articles met the requirements. For the index of serum HbA1c $\left(\mathrm{P}<0.00001 ; \mathrm{I}^{2}=98 \%\right)$ in patients with $\mathrm{DM}$ complicated with IDA, data were combined between studies using the random effects model, and the results showed that compared with the control group, there was a statistically substantial difference in the content of HbAlc between the two groups ( $\mathrm{MD}=0.84 ; 95 \% \mathrm{CI}: 0.06$ to $1.61 ; \mathrm{P}=0.04$ ), as shown in Figure 6.

Using the serum HbA1c of diabetic patients with IDA as the index, the results of the inverted funnel plot showed that the scattered points of the participants were roughly funneled downward. On the horizontal axis, they are arranged roughly symmetrically, which suggested that publication bias was not obvious, as shown in Figure 7.

\section{Discussion}

HbA1c is the product of non-enzymatic glycosylation between the free amino group of $\mathrm{Hb}$ and glucose (32). The $\mathrm{n}$-terminal valine amino of the $\beta$ chain of $\mathrm{Hb}$ is most exposed to plasma glucose, and its binding with glucose is an important component of glycosylated hemoglobin, known as HbA1c (33). The survival time of red blood cells in the human body is about 120 days, and HbA1c in the blood during erythropoiesis is maintained at a certain level, so the HbA1c can be 3 to 4 months before blood plasma glucose average, and the time of blood collection, blood collection state, and other factors, such as whether fasted 


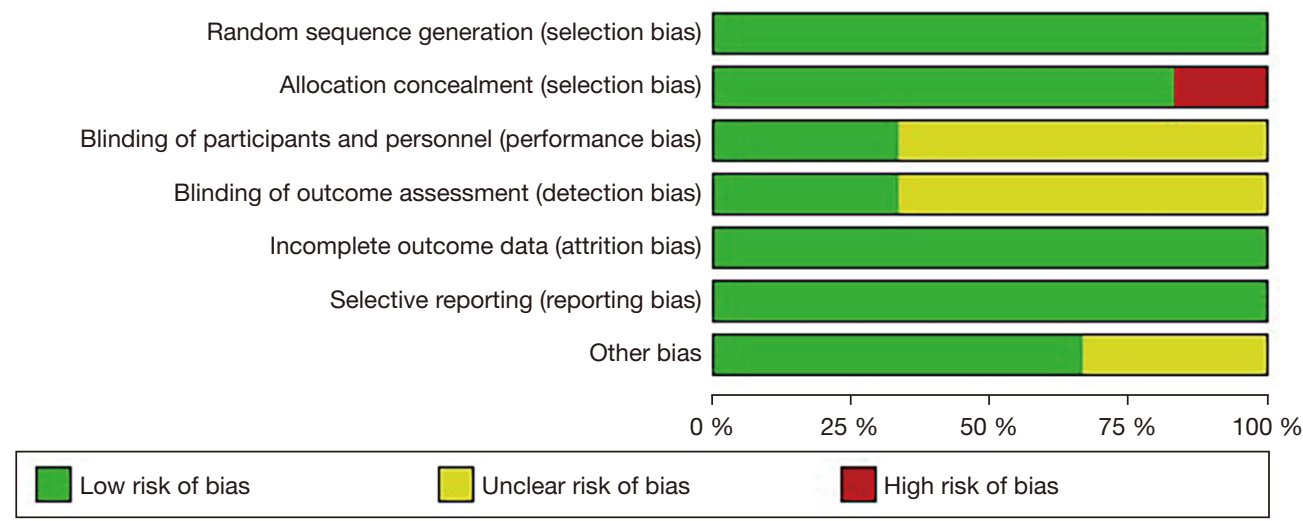

Figure 3 The bias evaluation bar graph of the included articles.

Table 2 Quality evaluation of the included literature

\begin{tabular}{|c|c|c|c|c|c|c|c|}
\hline First author & $\begin{array}{l}\text { Representativeness } \\
\text { of cases }\end{array}$ & $\begin{array}{l}\text { Control } \\
\text { selection }\end{array}$ & $\begin{array}{c}\text { Definition of } \\
\text { contrast }\end{array}$ & Comparability & $\begin{array}{l}\text { Methodologies for } \\
\text { leak investigation } \\
\text { and assessment }\end{array}$ & $\begin{array}{l}\text { Whether the investigation } \\
\text { methods of the two groups } \\
\text { are appropriate }\end{array}$ & $\begin{array}{l}\text { Total } \\
\text { score }\end{array}$ \\
\hline Shanthi B (27) & Yes & No & Yes & Yes & Yes & Yes & 5 \\
\hline Silva JF (28) & Yes & Yes & Yes & Yes & Yes & Yes & 7 \\
\hline Christy AL (30) & Yes & No & No & Yes & No & Yes & 8 \\
\hline Urrechaga E (31) & Yes & Yes & Yes & Yes & Yes & Yes & 6 \\
\hline
\end{tabular}

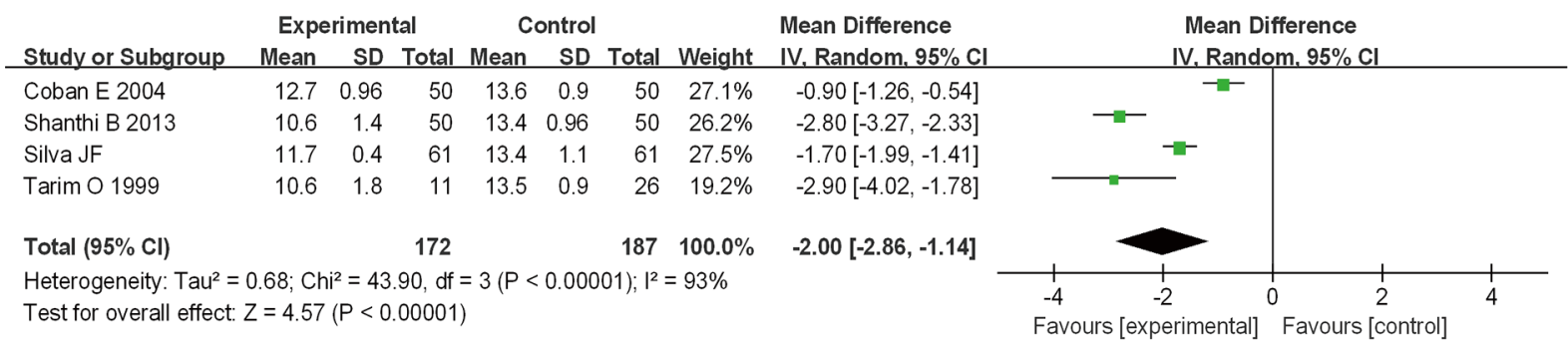

Figure 4 Forest plot for the serum $\mathrm{Hb}$ in diabetic patients with IDA. Hb, hemoglobin; IDA, iron deficiency anemia.

or not has nothing to do with its reading, therefore, it can reflect the mean value of long-term plasma glucose in diabetic patients $(34,35)$. The current detection methods of HbA1c can be divided into one item according to the difference in the structure of HbA1c, such as immunoassay, affinity chromatography, ion capture, and so on (36). At present, HbA1c is not only an observation index of mean plasma glucose in diabetic patients, but has also been used in the diagnosis of diabetes in European and American countries. The use of HbA1c has greatly increased, mainly because its determination does not require fasting and the intra-individual variability is relatively low (37). Diseases affecting the life span of $\mathrm{Hb}$ are thought to affect the results of blood A1c measurement. Iron deficiency anemia (IDA), the most common anemia disease, has a wide range of patients. Whether the results of blood A1c measurement 


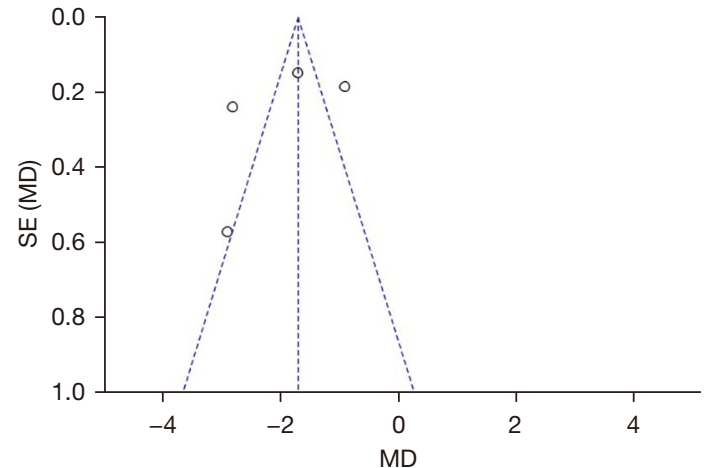

Figure 5 Funnel plot for the serum $\mathrm{Hb}$ in diabetic patients with IDA. Hb, hemoglobin; IDA, iron deficiency anemia.

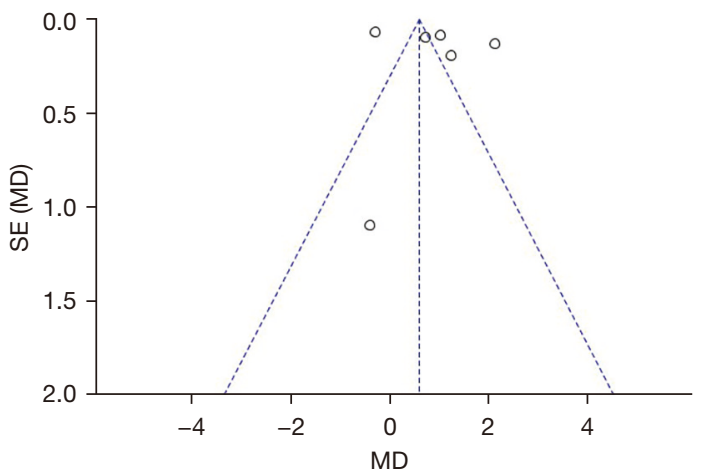

Figure 7 Funnel plot of serum HbA1c in nondiabetic patients with IDA. HbA1c, glycosylated hemoglobin; IDA, iron deficiency anemia.

\begin{tabular}{|c|c|c|c|c|c|c|c|}
\hline \multirow[b]{2}{*}{ Study or Subgroup } & \multicolumn{3}{|c|}{ Experimental } & \multicolumn{2}{|c|}{ Control } & \multirow[b]{2}{*}{ Total } & \multirow[b]{2}{*}{ Weight } \\
\hline & Mean & $S D$ & Total & Mean & SD & & \\
\hline Christy AL 2014 & 6.87 & 1.4 & 70 & 5.65 & 0.69 & 50 & $18.0 \%$ \\
\hline Coban E 2004 & 6.2 & 0.6 & 50 & 5.2 & 0.2 & 50 & $18.6 \%$ \\
\hline Shanthi B 2013 & 7.6 & 0.5 & 50 & 5.5 & 0.8 & 50 & $18.4 \%$ \\
\hline Silva JF & 5.3 & 0.4 & 61 & 5.6 & 0.4 & 61 & $18.7 \%$ \\
\hline Tarim O 1999 & 9.9 & 2.6 & 11 & 10.3 & 3.9 & 26 & $7.7 \%$ \\
\hline Urrechaga E 2018 & 8.5 & 1.5 & 410 & 7.8 & 1.3 & 357 & $18.6 \%$ \\
\hline Total $(95 \% \mathrm{Cl})$ & & & 652 & & & 594 & $100.0 \%$ \\
\hline $\begin{array}{l}\text { Heterogeneity: } \text { Tau }^{2} \\
\text { Test for overall effec }\end{array}$ & $\begin{array}{l}84 ; 0 \\
=?\end{array}$ & $=3$ & $\begin{array}{l}59, \\
4)\end{array}$ & 5( & & 1); & $=98 \%$ \\
\hline
\end{tabular}

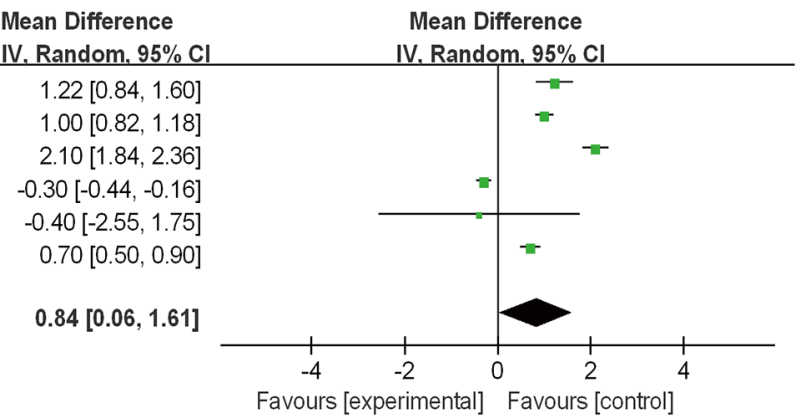

Figure 6 Forest plot of serum HbA1c in diabetic patients with IDA. HbA1c, glycosylated hemoglobin; IDA, iron deficiency anemia.

are accurate for these patients with iron deficiency anemia accurate, Whether the use of HbA1c to assess blood glucose levels in this group of patients is unclear, so further studies are needed to determine whether IDA has an impact on blood HbA1c measurements (38).

In summary, for patients with IDA, the possibility of a false increase in HbA1c must be taken into account when interpreting the clinical results, and should be supplemented by other tests, such as HbA1c. For patients with abnormal HbA1c results, clinicians should comprehensively consider the interfering factors of HbAlc measurement, conduct a comprehensive analysis of the results, and perform relevant necessary examinations to check for anemia and the type of anemia. Caution should be exercised before changing treatment, as anemia may exaggerate a patient's blood sugar status.

\section{Conclusions}

In this paper, in order to explore the IDA in patients with diabetes, we explored the effect of serum concentration of HbA1c in patients with diabetic and non-diabetic patients. To test the serum levels of the effect of HbAlc, detection methods, including immune method and ion-exchange chromatography were analyzed using meta-analysis. A total of 8 articles were included in the meta-analysis, and the results indicated that in the non-diabetic population, IDA can falsely increase the level of HbA1c. The mechanism of the increase of $\mathrm{HbAlc}$ in IDA requires further study.

\section{Acknowledgments}

Funding: The study was supported by Sichuan Science and Technology Program (2020YFS0434).

\section{Footnote}

Reporting Checklist: The authors have completed the PRISMA reporting checklist. Available at https://dx.doi. org/10.21037/apm-21-2944 
Conflicts of Interest: All authors have completed the ICMJE uniform disclosure form (available at https://dx.doi. org/10.21037/apm-21-2944). The authors have no conflicts of interest to declare.

Ethical Statement: The authors are accountable for all aspects of the work in ensuring that questions related to the accuracy or integrity of any part of the work are appropriately investigated and resolved.

Open Access Statement: This is an Open Access article distributed in accordance with the Creative Commons Attribution-NonCommercial-NoDerivs 4.0 International License (CC BY-NC-ND 4.0), which permits the noncommercial replication and distribution of the article with the strict proviso that no changes or edits are made and the original work is properly cited (including links to both the formal publication through the relevant DOI and the license). See: https://creativecommons.org/licenses/by-nc-nd/4.0/.

\section{References}

1. Palumbo C, Nicolaci N, La Manna AA, et al. Association between central diabetes insipidus and type 2 diabetes mellitus. Medicina (B Aires) 2018;78:127-30.

2. Karaa A, Goldstein A. The spectrum of clinical presentation, diagnosis, and management of mitochondrial forms of diabetes. Pediatr Diabetes 2015;16:1-9.

3. Patti G, Ibba A, Morana G, et al. Central diabetes insipidus in children: Diagnosis and management. Best Pract Res Clin Endocrinol Metab 2020;34:101440.

4. Garrahy A, Thompson CJ. Management of central diabetes insipidus. Best Pract Res Clin Endocrinol Metab 2020;34:101385.

5. Bastin M, Andreelli F. Corticosteroid-induced diabetes: Novelties in pathophysiology and management. Rev Med Interne 2020;41:607-16.

6. Riedel U, Schüßler E, Härtel D, et al. Wound treatment in diabetes patients and diabetic foot ulcers. Hautarzt 2020;71:835-42.

7. Grant PJ, Cosentino F, Marx N. Diabetes and coronary artery disease: not just a risk factor. Heart 2020;106:1357-64.

8. Fleeman L, Gostelow R. Updates in Feline Diabetes Mellitus and Hypersomatotropism. Vet Clin North Am Small Anim Pract 2020;50:1085-105.

9. Battarbee AN, Grant JH, Vladutiu CJ, et al. Hemoglobin A1c and Early Gestational Diabetes. J Womens Health
(Larchmt) 2020;29:1559-63.

10. Sun C, Wu C, Zhao W, et al. Glycosylated Hemoglobin A1c Predicts Intracerebral Hemorrhage with Acute Ischemic Stroke Post-Mechanical Thrombectomy. J Stroke Cerebrovasc Dis 2020;29:105008.

11. Orešković D, Raguž M, Predrijevac N, et al. Hemoglobin A1c in Patients with Glioblastoma-A Preliminary Study. World Neurosurg 2020;141:e553-8.

12. Tsapas A, Avgerinos I, Karagiannis T, et al. Comparative Effectiveness of Glucose-Lowering Drugs for Type 2 Diabetes: A Systematic Review and Network Metaanalysis. Ann Intern Med 2020;173:278-86.

13. Shibamoto J, Shoda K, Kubota T, et al. Prognostic impact of the preoperative hemoglobin A1c levels in patients with gastric cancer surgery depends on postoperative complications. Surg Today 2021;51:422-31.

14. Sookaromdee P, Wiwanitkit V. Estimated Blood Glucose and Hemoglobin A1C in COVID-19: a Preliminary Summarization. Clin Lab 2020. doi: 10.7754/Clin. Lab.2020.200421.

15. Pasricha SR, Tye-Din J, Muckenthaler MU, et al. Iron deficiency. Lancet 2021;397:233-48.

16. Garzon S, Cacciato PM, Certelli C, et al. Iron Deficiency Anemia in Pregnancy: Novel Approaches for an Old Problem. Oman Med J 2020;35:e166.

17. Sundararajan S, Rabe H. Prevention of iron deficiency anemia in infants and toddlers. Pediatr Res 2021;89:63-73.

18. Li N, Zhao G, Wu W, et al. The Efficacy and Safety of Vitamin C for Iron Supplementation in Adult Patients With Iron Deficiency Anemia: A Randomized Clinical Trial. JAMA Netw Open 2020;3:e2023644.

19. Mansour D, Hofmann A, Gemzell-Danielsson K. A Review of Clinical Guidelines on the Management of Iron Deficiency and Iron-Deficiency Anemia in Women with Heavy Menstrual Bleeding. Adv Ther 2021;38:201-25.

20. Dubey P, Thakur V, Chattopadhyay M. Role of Minerals and Trace Elements in Diabetes and Insulin Resistance. Nutrients 2020;12:1864.

21. Vaquero MP, Martínez-Maqueda D, Gallego-Narbón A, et al. Relationship between iron status markers and insulin resistance: an exploratory study in subjects with excess body weight. PeerJ 2020;8:e9528.

22. Liberal Â, Pinela J, Vívar-Quintana AM, et al. Fighting Iron-Deficiency Anemia: Innovations in Food Fortificants and Biofortification Strategies. Foods 2020;9:1871.

23. Cotter J, Baldaia C, Ferreira M, et al. Diagnosis and treatment of iron-deficiency anemia in gastrointestinal bleeding: A systematic review. World J Gastroenterol 
2020;26:7242-57.

24. Korom VG, Lueff S, Liposits A, et al. Is iron deficiency anemia always microcytic? Pol Arch Intern Med 2021;131:199-201.

25. Chopra VK, Anker SD. Anaemia, iron deficiency and heart failure in 2020: facts and numbers. ESC Heart Fail 2020;7:2007-11.

26. Coban E, Ozdogan M, Timuragaoglu A. Effect of iron deficiency anemia on the levels of hemoglobin A1c in nondiabetic patients. Acta Haematol 2004;112:126-8.

27. Shanthi B, Revathy C, Manjula Devi AJ, et al. Effect of iron deficiency on glycation of haemoglobin in nondiabetics. J Clin Diagn Res 2013;7:15-7.

28. Silva JF, Pimentel AL, Camargo JL. Effect of iron deficiency anaemia on HbAlc levels is dependent on the degree of anaemia. Clin Biochem 2016;49:117-20.

29. Tarim O, Küçükerdoğan A, Günay U, et al. Effects of iron deficiency anemia on hemoglobin A1c in type 1 diabetes mellitus. Pediatr Int 1999;41:357-62.

30. Christy AL, Manjrekar PA, Babu RP, et al. Influence of iron deficiency anemia on hemoglobin A1c levels in diabetic individuals with controlled plasma glucose levels. Iran Biomed J 2014;18:88-93.

31. Urrechaga E. Influence of iron deficiency on $\mathrm{Hb} \mathrm{A1c}$ levels in type 2 diabetic patients. Diabetes Metab Syndr 2018;12:1051-5.

32. Waheed H, Friedman H, Moin SF, et al. The Primary Structure of $\beta(\mathrm{I})$-Chain of Hemoglobin from Snake Sindhi Krait (Bungarus sindanus sindanus). Protein J

Cite this article as: Kuang L, Li W, Xu G, You M, Wu W, Li C. Systematic review and meta-analysis: influence of iron deficiency anemia on blood glycosylated hemoglobin in diabetic patients. Ann Palliat Med 2021;10(11):11705-11713. doi: 10.21037/ apm-21-2944
2016;35:193-201.

33. Akdi A, Ertem AG, Yayla Ç, et al. Glycosylated Hemoglobin A1c and Lipoprotein(a) in Patients Presenting With Premature Acute Coronary Syndrome. Angiology 2020;71:762.

34. Rayis DA, Ahmed ABA, Sharif ME, et al. Reliability of glycosylated hemoglobin in the diagnosis of gestational diabetes mellitus. J Clin Lab Anal 2020;34:e23435.

35. Doyle-Delgado K, Chamberlain JJ, Shubrook JH, et al. Pharmacologic Approaches to Glycemic Treatment of Type 2 Diabetes: Synopsis of the 2020 American Diabetes Association's Standards of Medical Care in Diabetes Clinical Guideline. Ann Intern Med 2020;173:813-21.

36. Heinemann L, Kaiser P, Freckmann G, et al. Higher HbA1c Measurement Quality Standards are Needed for Follow-Up and Diagnosis: Experience and Analyses from Germany. Horm Metab Res 2018;50:728-34.

37. Feig DS, Donovan LE, Zinman B, et al. Metformin in women with type 2 diabetes in pregnancy (MiTy): a multicentre, international, randomised, placebo-controlled trial. Lancet Diabetes Endocrinol 2020;8:834-44.

38. Goldenberg JZ, Day A, Brinkworth GD, et al. Efficacy and safety of low and very low carbohydrate diets for type 2 diabetes remission: systematic review and meta-analysis of published and unpublished randomized trial data. BMJ 2021;372:m4743.

(English Language Editor: J. Jones) 